\section{Discussion}

Acrodermatitis chronica atrophicans is a slow progressive disease that runs a biphasic course ${ }^{4}$ : the initial or inflammatory stage resembles erythema chronicum migrans, but is clearly differentiated by its diffuse or localised erythema. It usually affects one extremity and later spreads over its extensor surface. In this phase the histology of the skin shows infiltration by plasma cells, lymphocytes, and histiocytes. After periods varying from weeks to months, hyper- and hypopigmentation develop, and gradually the skin becomes frail like cigarette paper so that vessels and subcutaneous tissue become visible; on the other hand fibrosis and sclerosis also develop. In this late or atrophic stage biopsy specimens are pathognomonic showing the typical signs of epidermal atrophy, damaged and degenerated elastin and collagen, and dilated dermal vessels surrounded by plasma cell infiltrates.

In our patients the cutaneous lesions corresponded to the clinical picture of acrodermatitis chronica atrophicans, and the histological findings were compatible with the inflammatory stage of the disease. High antibody titres against $B$ burgdorferi (as found in both our cases) are diagnostic, ${ }^{3}$ the negative skin biopsy culture is not surprising, because isolation of the organism is notoriously difficult. $^{2}$

A history of tick bite (absent in case 2) is not mandatory in diagnosing diseases caused by $B$ burgdorferi because the bite is often overlooked or forgotten, and tabanids and mosquitoes may also harbour the pathogen..$^{5}$ Our patients had no other signs or symptoms of Lyme disease.

Benzylpenicillin has been reported to be effective in the inflammatory stage of acrodermatitis, but recommended treatment regimens differ. ${ }^{4}$ Because high doses of penicillin given parenterally have been successful in the later stages of Lyme disease, ${ }^{6}$ we prescribed intravenous benzylpenicillin 12 million units $/ \mathrm{m}^{2} /$ day for four weeks. Clinically this treatment was successful, although the skin changes had not completely disappeared at the time of writing. Raised antibody titres may persist for six to 12 months after treatment. ${ }^{3}$ As in late syphilis, it may be that the time taken to achieve seronegativity after acrodermatitis depends on the duration of the untreated infection.

Our observations show that acrodermatitis chronica atrophicans associated with $B$ burgdorferi may occur in childhood. Paediatricians should therefore be aware of this disorder because early diagnosis and treatment are important.

\section{References}

1 Steere AC, Malawista SE. Lyme disease. In: Mandell GL, Douglas RG, Bennet JE, eds. Principles and practice of infectious diseases. New York: John Wiley \& Sons, 1985: 1343-9.

2 Asbrink E, Hovmark A, Hederstedt B. The spirochetal etiology of acrodermatitis chronica atrophicans Herxheimer. Acta Derm Venereol (Stockh) 1984;64:506-12.

${ }^{3}$ Asbrink E, Hovmark A, Hederstedt B. Serologic studies of erythema chronicum migrans Afzelius and acrodermatitis chronica atrophicans with indirect immunofluorescence and enzyme-linked immunosorbent assays. Acta Derm Venereol (Stockh) 1985;65:509-14.

4 Burgdorf WHC, Worret W-I, Schultka O. Acrodermatitis chronica atrophicans. Int J Dermatol 1979;18:595-601.

${ }^{5}$ Magnarelli LA, Anderson JF, Barbour AG. The etiologic agent of Lyme disease in deer flies, horse flies, and mosquitoes. $J$ Infect Dis 1986;154:355-8.

6 Steere AC, Green J, Schoen RT, et al. Successful parenteral penicillin therapy of established Lyme arthritis. $N$ Engl J Med 1985;312:869-74.

Correspondence to Dr D Nadal, Department of Pediatrics, University of Zurich, Steinwiesstrasse $75, \mathrm{CH}-8032$ Zurich, Switzerland.

Received 15 June 1987

\title{
Blood counts in extremely low birthweight infants
}

\author{
N McINTOSH, C KEMPSON, AND R M TYLER
}

South West Thames Regional Neonatal Unit, St George's Hospital, London

SUMmary White blood, neutrophil, and platelet counts were higher in 101 infants with appropriate weight for gestational age than in 42 infants who were small for gestational age. The recognised postnatal rise in counts was seen in the infants of appropriate weight, but in the infants who were small for gestational age the counts fell for the first three days. 
The results of blood counts in extremely low birthweight infants $(<1000 \mathrm{~g})$ may lead to important decisions being taken about the treatment of probable infection, anaemia, or polycythaemia. We report here how extremely low birthweight infants who are either of an appropriate weight for gestational age or small for gestational age have intrinsically different blood counts. A knowledge of these differences may have implications for treatment.

\section{Patients and methods}

Blood counts measured during the first 14 days of life in 143 infants who were born consecutively and who weighed less than $1000 \mathrm{~g}$ were reviewed (101 of appropriate weight for gestational age and 42 who were small for gestational age). Arterial or venous sampling sites were used as we have previously shown that mean neutrophil counts were similar in blood drawn from these sites and mean haemoglobin concentrations were only slightly higher in venous blood specimens. ${ }^{1}$ Blood counts were estimated on a Coulter $S+4$ counter and the differential white count estimated from smears stained with Jenner-Geimsa. Unpaired $t$ tests were used to test the significance of differences between the two groups of babies.

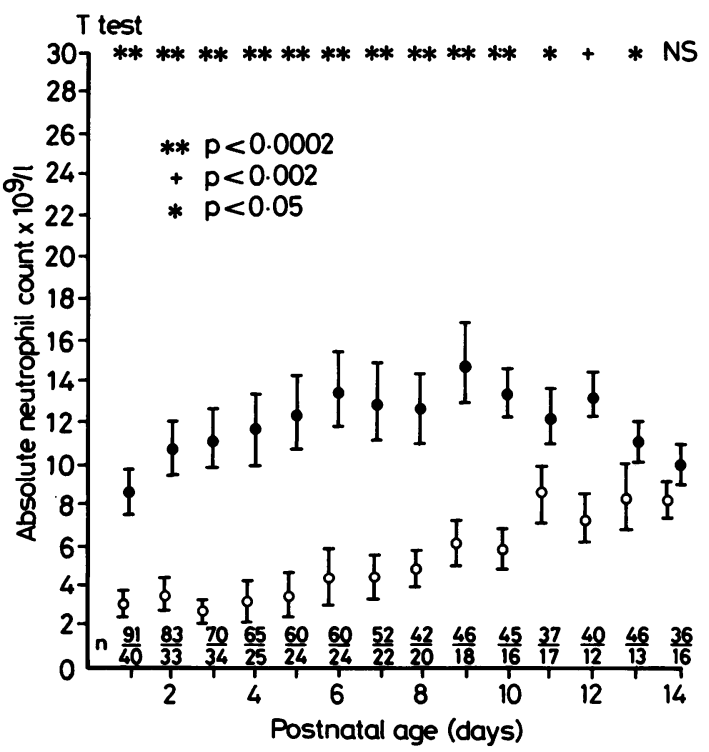

Fig 1 Absolute neutrophil count (SD) during the first 14 days of life in babies of appropriate weight for gestational age $(\mathrm{O})$ and babies who were small for gestational age $(\bigcirc)$.

\section{Results}

The mean haemoglobin concentration of the group who were small for gestational age at 24 hours $(161(\mathrm{SD} 25) \mathrm{g} / \mathrm{l})$ was higher than the group of infants with appropriate weight for gestational age (142 (27) $\mathrm{g} / \mathrm{l}, \mathrm{p}<0 \cdot 005)$. After this the results were confused by frequent blood transfusions. The mean corpuscular haemoglobin concentration of the group who were small for gestational age (326 (10) g/l) was lower than the group of appropriate weight (331 (18) $\mathrm{g} / \mathrm{l})$ on the first day $(\mathrm{p}<0.01)$ but the other red cell indices were similar in the two groups.

The mean neutrophil count of the group who were small for gestational age was significantly lower than the group of babies of appropriate weight until the 14th day of life (fig 1). The mean neutrophil count rose immediately after birth in the group of appropriate weight, but fell during the first three days of life in the group who were small for gestational age. In individual babies in this group the fall was so substantial that certain of them became neutropoenic $\left(<1 \times 10^{9} / 1\right)$. This finding occurred in the absence of infection and in infants in good general health. The mean platelet count of the group of infants who were small for gestational age was significantly lower than in the group of appropriate weight for the first 13 days of life (fig 2). In contrast

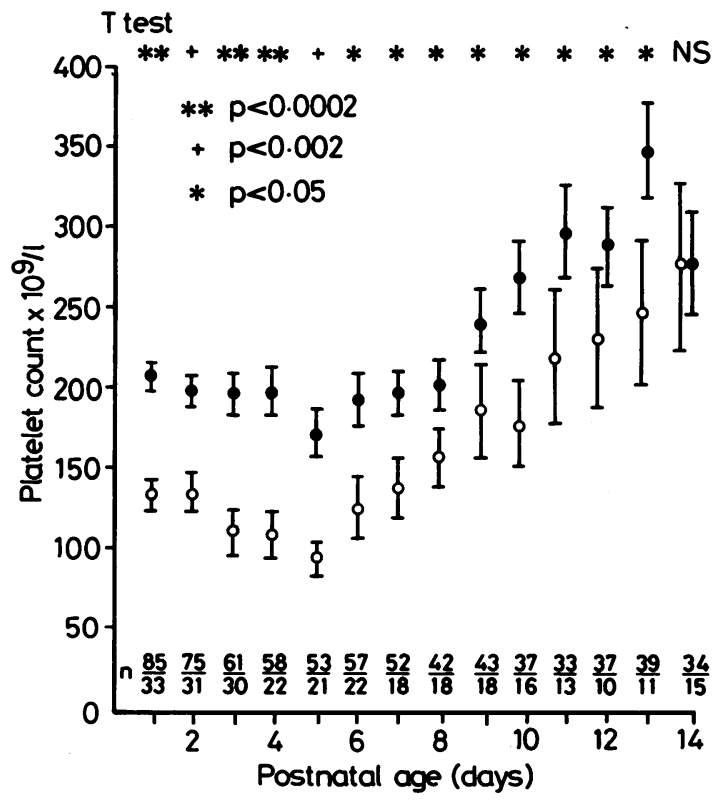

Fig 2 Platelet count (SD) during the first 14 days of life in babies of appropriate weight for gestational age (O) and babies who were small for gestational age (O). 
the mean (SD) nucleated red cell count of infants who were small for gestational age was considerably higher at birth $\left(260(276) v 53(94) \times 10^{9} / 1 ; \mathrm{p}<0.0001\right)$ and remained so for the first six days of life.

\section{Discussion}

Most infants of less than $1000 \mathrm{~g}$ birth weight are born vaginally after the spontaneous onset of preterm labour often associated with antepartum haemorrhage or premature rupture of membranes and they are of appropriate weight for gestational age. A minority are born by caesarean section because of severe toxaemia of pregnancy in the mother. The infants born to these mothers are frequently small for gestational age.

Mothers of these two groups of extremely low birthweight infants are likely to be intrinsically different, and they may have had different drug treatments. Mothers with toxaemia may show haemolysis, raised liver enzyme activities, and low platelet counts. $^{2}$ Brazy et al reported that babies born to mothers with severe hypertension had a high incidence of thrombocytopoenia, neutropoenia, and haemorrhagic complications compared with the babies born to normotensive controls who were retrospectively matched for length of gestation. ${ }^{3}$ Sibai et al, however, in a prospective pair controlled study were not able to confirm this. ${ }^{4}$ In neither of these studies was it possible to elaborate on whether the haematological findings were related to the underlying condition of the mother or the maternal drug treatment. Widerlöv et al suggested that maternal hydralazine treatment might cause neonatal thrombocytopoenia. ${ }^{5}$

Neutrophilia in the newborn may be seen in neonatal leukaemia where it is almost always associated with chromosome disorders. It has also been proposed that leukaemoid reactions that last for many days after birth may result from maternal steroid treatment. A recent study, however, comparing two matched groups of babies where one group of mothers had been treated with dexamethasone, was unable to show any haematological differences, and the only cases of very high white cell counts were seen in two infants whose mothers had not had steroids and in whom there was no evidence of infection (Thurlbeck and McIntosh, in preparation).

It is usually postulated that the higher haemoglobin concentration seen in infants who are small for gestational age is due to chronic intrauterine hypoxia. We know of no studies on the marrow of such infants, but it is possible that the lower white cell and platelet counts observed in these babies in the current study could be due to marrow stem cells being more committed in utero to producing cells of the red cell series in an attempt to improve oxygen transport and delivery in the fetus. At birth it may not be possible for the stem cells to switch immediately to white cell and platelet production. Some evidence supporting this theory is the significantly raised nucleated red cell count during the first six days of life in the group who were small for gestational age. It was only when the nucleated red cell count fell that the peripheral white count and platelet count rose.

The purpose of this paper is to draw attention to differences in the blood counts of these two groups of extremely low birthweight babies, not to account for them. We believe that the differences are so spectacular in the first week of life that it may be important to anticipate them. For example, babies who are small for gestational age often have profound neutropoenia, and despite the fact that they are born by caesarean section with no predisposition to infection it may be reasonable to give them prophylactic antibiotics in the same way as one would to other neutropoenic immunocompromised patients. One infant early in this study, who was born with a neutrophil count of $0.8 \times 10^{9} / 1$, suddenly developed a rapidly fatal septicaemia on the 4th day of life. In retrospect his neutrophil count had fallen steadily after birth and we believe that he developed septicaemia because of neutropoenia, not the reverse. We have not seen any complications directly resulting from the thrombocytopoenia but have on several occasions felt obliged to perform clotting screens to ensure that the thrombocytopoenia was not part of disseminated intravascular coagulation.

We would like to thank the staff of the neonatal unit for care of the infants and the staff of the haematology department for their excellent service to our unit. A full table of results is available from N McIntosh.

\section{References}

1 Thurlbeck SM, McIntosh N. Preterm blood counts vary with sampling site. Arch Dis Child 1987;62:74-5.

2 Weinstein L. Syndrome of haemolysis, elevated liver enzymes, and low platelet count: a severe consequence of hypertension in pregnancy. Am J Obstet Gynecol 1982;142:159-67.

3 Brazy JE, Grimm JK, Little VA. Neonatal manifestations of severe maternal hypertension occurring before the thirty sixth week of pregnancy. $J$ Pediatr 1982;100:265-71.

4 Sibai BM, Watson DL, Hill GA, Spinnato JE, Anderson G. Maternal-fetal correlations in patients with severe preeclampsia/eclampsia. Obstet Gynecol 1983;62:745-50.

5 Widerlöv E, Karlman I, Storsäter J. Hydralazine induced neonatal thrombocytopoenia. $N$ Engl J Med 1980;303:1235.

Correspondence to Professor N McIntosh, Department of Child Life and Health, 17 Hatton Place, Edinburgh EH9 1UW.

Received 7 July 1987 